\title{
Prognostic Factors for Visual Outcomes Following the First Episode of NMOSD-Related Optic Neuritis in Affected Eyes
}

This article was published in the following Dove Press journal: Clinical Ophthalmology

\author{
Watcharaporn Thongmee ${ }^{1}{ }^{\prime}$ \\ Tanyatuth Padungkiatsagul (D) ${ }^{\prime}$ \\ Panitha Jindahra ${ }^{2}$ \\ Chaiyos Khongkhatithum ${ }^{3}$ \\ Lunliya Thampratankul ${ }^{3}$ \\ Kavin Vanikieti ${ }^{\prime}$ \\ 'Department of Ophthalmology, Faculty \\ of Medicine Ramathibodi Hospital, \\ Mahidol University, Bangkok, Thailand; \\ ${ }^{2}$ Department of Medicine, Faculty of \\ Medicine Ramathibodi Hospital, Mahidol \\ University, Bangkok, Thailand; \\ ${ }^{3}$ Department of Pediatrics, Faculty of \\ Medicine Ramathibodi Hospital, Mahidol \\ University, Bangkok, Thailand
}

Objective: We aim to identify prognostic factors for visual outcomes following a first episode of neuromyelitis optica spectrum disorder-related optic neuritis (NMOSD-ON) in affected eyes.

Materials and Methods: This was a single-center, retrospective study. The study included 50 individuals who were diagnosed with NMOSD-ON (63 affected eyes) in a tertiary institution over a 12-year period. Data regarding any second, or higher, episodes of NMOSD-ON in each eye were not taken into consideration. Medical records of included individuals were reviewed. Demographic data, clinical and magnetic resonance imaging characteristics, and treatment outcomes were collected. Main outcome measures of the study were prognostic factors for good visual outcome (best-corrected visual acuity $(B C V A) \geq 20 / 200$ ) following an initial episode of NMOSD-ON in affected eyes.

Results: Sixty-three affected eyes of 50 individuals ( 3 men and 47 women) were included. BCVA at nadir that was better than counting fingers (CF) (odds ratio 10.43, 95\% confidence interval 1.04, 104.45, $p=0.046$ ) and time from NMOSD-ON onset to intravenous methylprednisolone (IVMP), less than 21 days (odds ratio 10.73, 95\% confidence interval 1.91, $60.01, p=0.007$ ), were significantly associated with good visual outcomes.

Conclusion: BCVA at nadir that was better than CF and treatment with IVMP within 21 days of symptom onset were important prognostic factors of good visual outcomes following a first episode of NMOSD-ON in affected eyes.

Keywords: optic neuritis, neuromyelitis optica spectrum disorder, prognostic factors, visual outcomes, Thai

\section{Introduction}

Neuromyelitis optica spectrum disorder (NMOSD) is a severe demyelinating disease. It is a spectrum of disease that includes optic neuritis (ON), longitudinally extensive transverse myelitis, area postrema, brainstem, diencephalon, and cerebral syndrome. One of the most-common presentations is acute-onset ON. Patients tend to present with severe visual loss, often accompanied by periorbital pain. Early treatment not only preserves better visual outcomes, but also prevents further morbidity and mortality.

NMOSD is a central nervous system (CNS) disease associated with aquaporin-4 (AQP4) antibodies. Initial treatment with high-dose intravenous methylprednisolone (IVMP, $1 \mathrm{gm}$ daily for 3 to 5 consecutive days) is recommended. ${ }^{1-3}$ Plasma exchange (PLEX) therapy is suggested for patients who have severe clinical attacks
Correspondence: Kavin Vanikieti Department of Ophthalmology, Faculty of Medicine Ramathibodi Hospital, Mahidol University, 270 Rama VI Road, Bangkok 10400, Thailand

Tel +662 20l 1526

Fax +6622012729

Email Vanikieti.kavin@gmail.com 
and who are unresponsive to glucocorticoids. ${ }^{1-3}$ Intravenous immunoglobulin has not been specifically evaluated for acute attacks and is rarely used in this setting. ${ }^{4}$ Only a few studies have identified prognostic factors regarding disease outcomes following treatment for NMOSD. Factors predictive of worse outcome include the number of relapses within the first 2 years, the severity of the first attack, older age at disease onset, length of the ON lesion, and perhaps an association with other autoimmune diseases, including autoimmune antibody status. ${ }^{5-9}$ In one retrospective study of $\mathrm{ON}$ overall (not exclusively neuromyelitis optica spectrum disorder-related optic neuritis (NMOSD-ON)), the researchers found that younger age at $\mathrm{ON}$ onset and good initial visual acuity were associated with good visual outcomes. ${ }^{10}$ However, the authors did not report the prognostic factors for visual outcomes following the first episode of NMOSD-ON per eye. Therefore, our study aimed to identify the prognostic factors for visual outcomes following the first episode of NMOSD-ON in affected eyes.

\section{Materials and Methods}

This study followed the tenets of the Declaration of Helsinki and was approved by the Institutional Review Board (IRB) of the Faculty of Medicine Ramathibodi Hospital, Mahidol University, Bangkok, Thailand. Patients were informed about the purpose of the study. Consent was obtained from the patients prior to study commencement. All patient data were kept confidentially in our database. Electronic medical records of 50 patients who were diagnosed with NMOSD$\mathrm{ON}$ in the Neuro-Ophthalmology clinic, Faculty of Medicine Ramathibodi Hospital, between January 2008 and June 2020, were retrospectively reviewed. For the diagnosis of NMOSD, we followed the international consensus diagnostic criteria for NMOSD with or without AQP4 antibodies. ${ }^{11}$ All AQP4 antibody testing was performed using cell-based assay. We included eyes that experienced at least one episode of NMOSD-ON. Only data regarding a first episode of NMOSD-ON in affected eyes were evaluated. Thus, data regarding any second, or higher, episodes of NMOSD-ON in each eye were not taken into consideration. Exclusion criteria included affected eyes with at least one of the following: incomplete medical data, or visually significant cataract and/or other ocular diseases affecting best-corrected visual acuity (BCVA).

The following data were collected from the patients' medical records: AQP4 serostatus, gender, age at first episode of $\mathrm{ON}$ in each affected eye, coexisting autoimmune disease, autoimmune antibody, concurrent clinical syndrome of NMOSD during first episode of $\mathrm{ON}$ in each affected eye, clinical characteristics including laterality, presence of pain, BCVA at nadir and presence of a swollen optic disc. Anterior visual pathway (AVP) and CNS magnetic resonance imaging (MRI) performed during the first episode of NMOSD-ON in each eye were also evaluated. Each AVP scan was composed of five segments: orbital optic nerve, intracanalicular optic nerve, intracranial optic nerve, optic chiasm and optic tract. ${ }^{12}$ (Figure 1) Treatment outcomes, including time from initial ocular symptoms (pain and/or visual loss) to IVMP administration, and BCVA at recovery were also analysed.

The primary goal of our study was to identify factors prognostic of good visual outcome following only the first episode of NMOSD-ON in each affected eye. A "good visual outcome" was defined as BCVA at recovery of equal to, or better than $20 / 200$ in the affected eye. For individuals with bilateral simultaneous involvement during their first episode of NMOSD-ON, medical data for the two eyes were analysed independently.

\section{Statistical Analysis}

Categorical data were summarized using proportions and percentages, and analysed using a Chi-square test or Fisher's exact test, as appropriate. Continuous data were summarized using medians and ranges, due to the non-normal distribution of the data, and analysed using the twosample $t$-test or Mann-Whitney U-test, depending on the distribution of data. Univariate and multivariate logistic regression analyses were used to evaluate prognostic factors. Snellen VA records were converted to the logarithm of the minimum angle of resolution (logMAR) for statistical analysis. BCVA of counting fingers (CF), hand motion, light perception and no light perception were converted to 2.6, 2.7, 2.8 and $2.9 \log$ MAR, respectively. ${ }^{13,14}$ Statistical analyses were performed using STATA software, version 16.0 (StataCorp LLC, TX, USA). $P$-values less than 0.05 were considered statistically significant.

\section{Results}

\section{Demographics and Clinical Characteristics}

Sixty-three eyes of 50 individuals ( 3 men and 47 women) were included in the study; all were Asian. The female: male ratio was 15.6: 1. AQP4 antibodies were positive in 45 of 50 patients (90\%). MOG (myelin oligodendrocyte glycoprotein) antibodies were tested in all AQP4 seronegative patients (5 

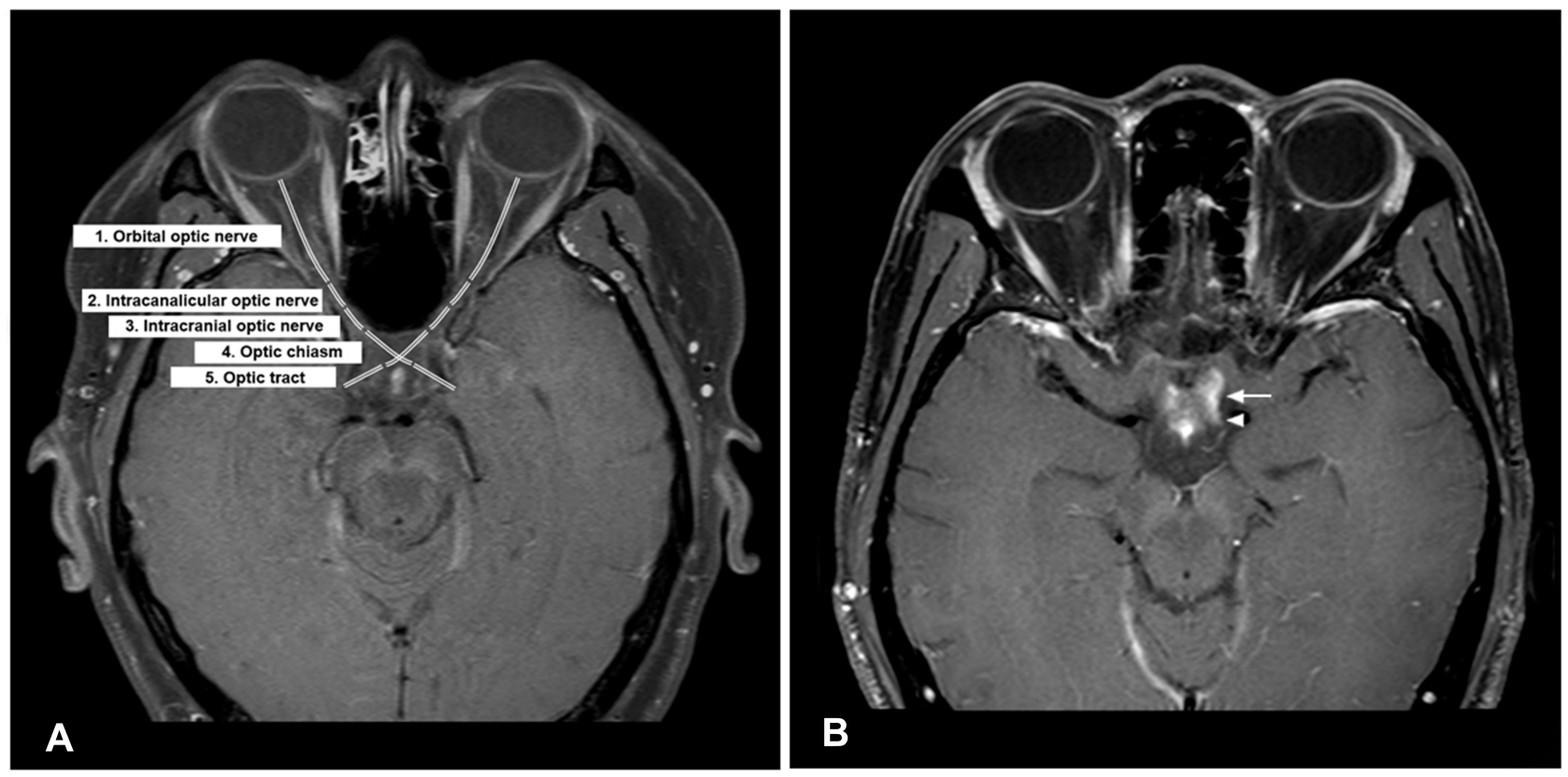

Figure I (A) Each AVP scan was composed of five segments: orbital optic nerve, intracanalicular optic nerve, intracranial optic nerve, optic chiasm and optic tract. (B) Fatsuppressed TI-weighted with gadolinium contrast axial MRI showing enhancement of optic chiasm (arrow) and optic tract (arrowhead).

patients). None of these 5 patients demonstrated MOG antibodies. In our study, pediatric patients were individuals who were less than 18 years of age. We included 12 affected eyes ( $19 \%$ of total affected eyes) of 6 pediatric patients $(12 \%$ of all patients). The median age at the first episode of NMOSD-ON in each affected eye was 36 years (range 4-84 years). There were 5 individuals with systemic lupus erythematosus, 4 with Sjogren syndrome and 2 with hyperthyroidism in our cohort. Myelitis, which was discovered concurrently in 10 eyes $(15.8 \%)$, was the most-common clinical syndrome of NMOSD during the initial ON episodes. Eight patients ( $16 \%$ of all patients) presented with bilateral simultaneous ON (16 affected eyes). The other 42 patients (84\% of all patients) presented with unilateral $\mathrm{ON}$ (42 affected eyes). During follow-up, 5 patients out of these 42 patients developed $\mathrm{ON}$ in the contralateral eyes (previously non-ON eyes) (5 affected eyes). Thus, a total of 63 affected eyes were included in our study. Most of the affected eyes (37 eyes, $58.6 \%$ ) had BCVA at nadir $<20 / 400$, and 4 of them had no perception of light. Pain and a swollen optic disc were present in 36 eyes $(57.1 \%)$ and 18 eyes (28.5\%), respectively. Demographic data and clinical characteristics are summarized in Table 1.

\section{MRI Characteristics}

AVP images were available for 57 affected eyes. Thirty-eight eyes $(66.6 \%)$ showed 2 or more consecutive segments of AVP involvement. The orbital optic nerve, involved in 45 eyes (78.9\%), was the most-affected segment. CNS MRI scans were available for 61 affected eyes. CNS MRI abnormalities were observed in the scans associated with 31 eyes $(50.8 \%)$. MRI characteristics from the first episode of NMOSD-ON in each affected eye are summarized in Table 2.

\section{Treatment Outcomes}

All patients in this cohort were treated with 3-5 consecutive days of intravenous methylprednisolone (IVMP) $1 \mathrm{~g} /$ day (for adults) or $30 \mathrm{mg} / \mathrm{kg} /$ day (maximum $1 \mathrm{~g} /$ day) (for pediatrics), followed by slowly tapered prednisolone for 2-3 months and immunosuppressive drugs.

The median time from initial ocular symptoms (pain and/or visual loss) to the start of IVMP administration was 4 days (range 1-60 days). The duration of IVMP treatment ranged from 3 to 5 days. After acute treatment with IVMP, nearly all affected eyes (95.2\%) showed improved BCVA at recovery, in a median time of 56 days (range 7-196 days). Median BCVA at recovery was $0.3 \log$ MAR (range 0.0-2.7), which was significantly better than the median BCVA at nadir (2.6 logMAR, range 0.18-2.9, $p<0.001$ ). Adjunct PLEX therapy was performed in connection with only 4 of the affected eyes $(6.35 \%)$, which did not respond to IVMP. Half of those eyes showed improvement in BCVA at recovery. The treatment outcomes following the first episode of NMOSD-ON are summarized in Table 3. 
Table I Demographic Data and Clinical Characteristics

\begin{tabular}{|c|c|}
\hline Variables & Values \\
\hline Number of patients & 50 \\
\hline Number of affected eyes & 63 \\
\hline \multicolumn{2}{|l|}{ AQP4 serostatus } \\
\hline - AQP4 positive, $n$ of patients (\%) & 45 (90\%) \\
\hline - AQP4 negative, $\mathrm{n}$ of patients (\%) & $5(10 \%)$ \\
\hline \multicolumn{2}{|l|}{ Gender } \\
\hline - Male, $\mathrm{n}$ of patients (\%) & $3(6 \%)$ \\
\hline - Female, $\mathrm{n}$ of patients (\%) & 47 (94\%) \\
\hline $\begin{array}{l}\text { Age at first episode of } \mathrm{ON} \text { in each affected eye (years), } \\
\text { median (min, max) }\end{array}$ & $36(4,84)$ \\
\hline Co-existing autoimmune disease, $\mathrm{n}$ of patients (\%) & II (22\%) \\
\hline - SLE, $\mathrm{n}$ of patients (\%) & $5(10 \%)$ \\
\hline - Sjogren syndrome, $\mathrm{n}$ of patients (\%) & $4(8 \%)$ \\
\hline - Hyperthyroidism, $\mathrm{n}$ of patients (\%) & $2(4 \%)$ \\
\hline Autoimmune antibody, $\mathrm{n}$ of patients (\%) & $26(52 \%)$ \\
\hline $\begin{array}{l}\text { Concurrent clinical syndrome of NMOSD during first } \\
\text { episode of ON in each eye, } n \text { of eyes (\%) }\end{array}$ & $15(23.8 \%)$ \\
\hline - Myelitis, $\mathrm{n}$ of eyes (\%) & $10(15.8 \%)$ \\
\hline - Area postrema syndrome, $\mathrm{n}$ of eyes (\%) & $2(3.2 \%)$ \\
\hline - Myelitis with area postrema syndrome, $\mathrm{n}$ of eyes (\%) & $2(3.2 \%)$ \\
\hline - Myelitis with brainstem syndrome, $\mathrm{n}$ of eyes (\%) & $\mathrm{I}(\mathrm{I} .6 \%)$ \\
\hline \multicolumn{2}{|l|}{ Laterality of first $\mathrm{ON}$ presentation } \\
\hline - Unilateral, n of patients (\%) & $42(84 \%)$ \\
\hline - Bilateral simultaneous, $\mathrm{n}$ of patients (\%) & $8(16 \%)$ \\
\hline $\begin{array}{l}\text { Presence of pain at first episode of } O N \text { in each affected } \\
\text { eye, } n \text { of eyes (\%) }\end{array}$ & $36(57.1 \%)$ \\
\hline BCVA at nadir (logMAR), median (min, max) & $2.6(0.18,2.9)$ \\
\hline \multicolumn{2}{|l|}{ BCVA at nadir } \\
\hline - $20 / 20$ to $20 / 70, \mathrm{n}$ of eyes $(\%)$ & $7(11.2 \%)$ \\
\hline$\bullet<20 / 70$ to $20 / 200, \mathrm{n}$ of eyes (\%) & $12(19.0 \%)$ \\
\hline - $<20 / 200$ to $20 / 400, n$ of eyes (\%) & $7(11.2 \%)$ \\
\hline - $<20 / 400$ to light perception, $n$ of eyes (\%) & $33(52.3 \%)$ \\
\hline - No perception of light, $n$ of eyes (\%) & $4(6.3 \%)$ \\
\hline Presence of swollen optic disc, $\mathrm{n}$ of eyes (\%) & $18(28.5 \%)$ \\
\hline
\end{tabular}

Abbreviations: $n$, number; min, minimum; max, maximum; AQP4, aquaporin-4; ON, optic neuritis; SLE, systemic lupus erythematosus; logMAR, logarithm of the minimum angle of resolution; BCVA, best-corrected visual acuity.

\section{Prognostic Factors Analysis of Good Visual Outcome}

In our study, a good visual outcome was defined as BCVA at recovery following the first episode of NMOSD-ON as equal to, or better than $20 / 200$ in the affected eye. Analysis of factors prognostic of good visual outcome is provided in Table 4. Univariate and multivariate logistic regression analyses (Tables 5 and 6, respectively) showed that the BCVA at nadir was significantly related to a good visual outcome
Table 2 MRI Characteristics During the First Episode of NMOSD-ON in Each Affected Eye

\begin{tabular}{|l|l|}
\hline Variables & Values \\
\hline $\begin{array}{l}\text { Segmental enhancement of AVP, } n \text { of eyes with } \\
\text { enhancement (percentages of the } 57 \text { eyes with } \\
\text { available AVP images) }\end{array}$ \\
- Orbital optic nerve & \\
- Intracanalicular optic nerve & $45(78.9 \%)$ \\
- Intracranial optic nerve & $40(70.1 \%)$ \\
- Optic chiasm & $22(38.6 \%)$ \\
- Optic tract & $6(10.5 \%)$ \\
- $\geq 2$ consecutive segments & $3(5.2 \%)$ \\
\hline CNS MRI abnormality, $n$ of eyes with abnormality & $38(66.6 \%)$ \\
(percentages of the 6I eyes with available CNS & \\
MRI images) & \\
- Brain lesion only & $21(30.8 \%)$ \\
- Cord lesion only & $4(6.5 \%)$ \\
- Both brain and cord lesions & $6(9.8 \%)$ \\
\hline
\end{tabular}

Abbreviations: MRI, magnetic resonance imaging; NMOSD-ON, neuromyelitis optica spectrum disorder-related optic neuritis; n, number; AVP, anterior visual pathway; CNS, central nervous system.

Table 3 Treatment Outcomes Following the First Episode of NMOSD-ON in Each Affected Eye

\begin{tabular}{|c|c|}
\hline Variables & Values \\
\hline $\begin{array}{l}\text { Time from initial ocular symptoms (pain and/or visual loss) to } \\
\text { start of IVMP (days), median (min, max) }\end{array}$ & $4(1,60)$ \\
\hline Duration of IVMP therapy (days), median (min, max) & $5(3,5)$ \\
\hline BCVA at recovery (logMAR), median (min, max) & $0.3(0,2.7)$ \\
\hline $\begin{array}{l}\text { BCVA at recovery } \\
\text { - } 20 / 20 \text { to } 20 / 70, n \text { of eyes (\%) } \\
\text { - }<20 / 70 \text { to } 20 / 200, n \text { of eyes (\%) } \\
\text { - }<20 / 200 \text { to } 20 / 400, n \text { of eyes (\%) } \\
\text { - }<20 / 400 \text { to light perception, } n \text { of eyes (\%) } \\
\text { - No perception of light, } n \text { of eyes (\%) }\end{array}$ & $\begin{array}{l}42(66.6 \%) \\
12(19.0 \%) \\
2(3.2 \%) \\
7(11.2 \%) \\
0\end{array}$ \\
\hline $\begin{array}{l}\text { Visual outcome (BCVA) } \\
\text { - Improved, } n \text { of eyes (\%) } \\
\text { - Stable, } n \text { of eyes (\%) } \\
\text { - Worsened, } n \text { of eyes (\%) }\end{array}$ & $\begin{array}{l}60(95.2 \%) \\
2(3.2 \%) \\
1(1.6 \%)\end{array}$ \\
\hline Time to BCVA recovery (days), median (min, max) & $56(7,196)$ \\
\hline
\end{tabular}

Abbreviations: NMOSD-ON, neuromyelitis optica spectrum disorder-related optic neuritis; IVMP, intravenous methylprednisolone; min, minimum; max, maximum; logMAR, logarithm of the minimum angle of resolution; $n$, number; BCVA, best-corrected visual acuity.

when the BCVA at nadir was better than $\mathrm{CF}$ (odds ratio (OR) 10.43, 95\% confidence interval (CI) 1.04, 104.45, $p=$ 0.046). Regarding the time from initial ocular symptoms to IVMP, a good visual outcome was significantly more likely in cases treated with IVMP within 21 days of NMOSD-ON onset (OR 10.73, 95\% CI 1.91, 60.01, $p=0.007)$. 
Table 4 Analysis of Factors Prognostic of Good Visual Outcome Following the First Episode of NMOSD-ON in Each Affected Eye

\begin{tabular}{|c|c|c|c|}
\hline Prognostic Factors & $\begin{array}{l}\text { BCVA at Recovery } \geq \\
20 / 200 \\
(\mathrm{~N}=54 \text { Eyes })\end{array}$ & $\begin{array}{l}\text { BCVA at Recovery < } \\
20 / 200 \\
(\mathrm{~N}=9 \text { Eyes })\end{array}$ & $\begin{array}{l}\mathrm{p}- \\
\text { value }\end{array}$ \\
\hline \multicolumn{4}{|l|}{ Demographic data } \\
\hline \multicolumn{4}{|l|}{ AQP4 serostatus } \\
\hline - $\mathrm{AQP4}$ positive, $\mathrm{n}$ of eyes (\%) & $46(85.2 \%)$ & $9(100 \%)$ & 0.59 \\
\hline - $\mathrm{AQP4}$ negative, $\mathrm{n}$ of eyes (\%) & $8(14.8 \%)$ & 0 & \\
\hline \multicolumn{4}{|l|}{ Gender } \\
\hline - Male, $\mathrm{n}$ of eyes $(\%)$ & $5(9.3 \%)$ & 0 & 1.00 \\
\hline - Female, $\mathrm{n}$ of eyes (\%) & $49(90.7 \%)$ & $9(100 \%)$ & \\
\hline Age at first episode of $\mathrm{ON}$ in each affected eye (years), median (min, max) & $37(4,84)$ & $29(4,80)$ & 0.85 \\
\hline Co-existing autoimmune disease, $\mathrm{n}$ of eyes (\%) & II (20.3\%) & $2(22.2 \%)$ & 1.00 \\
\hline - SLE, $n$ of eyes $(\%)$ & $6(11.1 \%)$ & I (II.1\%) & 1.00 \\
\hline - Sjogren syndrome, $\mathrm{n}$ of eyes (\%) & $4(7.4 \%)$ & 0 & 1.00 \\
\hline - Hyperthyroidism, $\mathrm{n}$ of eyes (\%) & $\mathrm{I}(1.8 \%)$ & I (II.1\%) & 0.26 \\
\hline Autoimmune antibody, $n$ of eyes (\%) & $27(50 \%)$ & $3(33.3 \%)$ & 0.47 \\
\hline $\begin{array}{l}\text { Concurrent clinical syndrome of NMOSD during first episode of ON in each affected eye, } n \text { of eyes } \\
\text { (\%) }\end{array}$ & $12(22.2 \%)$ & $3(33.3 \%)$ & 0.43 \\
\hline - Myelitis, $\mathrm{n}$ of eyes (\%) & $8(14.8 \%)$ & $2(22.2 \%)$ & 0.62 \\
\hline - Area postrema syndrome, $\mathrm{n}$ of eyes (\%) & I (I.8\%) & I (II.1\%) & 0.26 \\
\hline - Myelitis with area postrema syndrome, $\mathrm{n}$ of eyes (\%) & $2(3.8 \%)$ & 0 & 1.00 \\
\hline - Myelitis with brainstem syndrome, $n$ of eyes (\%) & $\mathrm{I}(1.8 \%)$ & 0 & 1.00 \\
\hline \multicolumn{4}{|l|}{ Clinical characteristics } \\
\hline \multicolumn{4}{|l|}{ Laterality at first episode of $\mathrm{ON}$ in each affected eye } \\
\hline - Unilateral, $\mathrm{n}$ of eyes (\%) & $4 \mid(76 \%)$ & $6(66.6 \%)$ & 0.68 \\
\hline - Bilateral simultaneous, $\mathrm{n}$ of eyes (\%) & $13(24 \%)$ & $3(33.3 \%)$ & \\
\hline Presence of pain at first episode of $\mathrm{ON}$ in each affected eye, $\mathrm{n}$ of eyes (\%) & $33(61.1 \%)$ & $3(33.3 \%)$ & 0.15 \\
\hline BCVA at nadir (logMAR), median (min, max) & $2.21(0.18,2.9)$ & $2.6(0.7,2.7)$ & 0.19 \\
\hline BCVA at nadir better than CF, $\mathrm{n}$ of eyes (\%) & $27(50 \%)$ & I (II.1\%) & $0.03^{*}$ \\
\hline Presence of swollen optic disc, $\mathrm{n}$ of eyes (\%) & $14(25.9 \%)$ & $4(44.4 \%)$ & 0.26 \\
\hline \multicolumn{4}{|l|}{ MRI characteristics ${ }^{\dagger}$} \\
\hline \multirow{2}{*}{\multicolumn{4}{|c|}{$\begin{array}{l}\text { Segmental enhancement of AVP, [n of eyes with enhancement/n of eyes with available AVP images per } \\
\text { BCVA at recovery group] }\end{array}$}} \\
\hline & \\
\hline - Orbital optic nerve & $36 / 48(75 \%)$ & $9 / 9(100 \%)$ & 0.18 \\
\hline - Intracanalicular optic nerve & $35 / 48(72.9 \%)$ & $5 / 9(55.5 \%)$ & 0.42 \\
\hline - Intracranial optic nerve & $19 / 48(39.5 \%)$ & $3 / 9(33.3 \%)$ & 1.00 \\
\hline - Optic chiasm & $6 / 48(12.5 \%)$ & 0 & 0.57 \\
\hline - Optic tract & $3 / 48(6.25 \%)$ & 0 & 1.00 \\
\hline - $\geq 2$ consecutive segments & $33 / 48(68.7 \%)$ & $5 / 9(55.5 \%)$ & 0.46 \\
\hline $\begin{array}{l}\text { CNS MRI abnormality, [n of eyes with abnormality/n of eyes with available CNS MRI images per BCVA } \\
\text { at recovery group] }\end{array}$ & $27 / 52(51.9 \%)$ & $4 / 9(44.4 \%)$ & 0.73 \\
\hline - Brain lesion only & $19 / 52(36.5 \%)$ & $2 / 9(22.2 \%)$ & 0.47 \\
\hline - Cord lesion only & $3 / 52(5.8 \%)$ & $1 / 9(11.1 \%)$ & 0.48 \\
\hline - Both brain and cord lesions & $5 / 52(9.6 \%)$ & $1 / 9(11.1 \%)$ & 1.00 \\
\hline \multicolumn{4}{|l|}{ IVMP treatment } \\
\hline \multicolumn{4}{|l|}{ Time from initial ocular symptoms (pain and/or visual loss) to start of IVMP } \\
\hline - Days, median (min, max) & $4(1,42)$ & $21(1,60)$ & $0.04 *$ \\
\hline - Less than $2 \mathrm{I}$ days, $\mathrm{n}$ of eyes (\%) & $47(87 \%)$ & $4(44.4 \%)$ & $0.003 *$ \\
\hline Duration of IVMP treatment (days), median (min, max) & $4(3,5)$ & $5(3,5)$ & 0.72 \\
\hline Time to BCVA recovery (days), median (min, max) & $56(7,196)$ & $45.5(14,98)$ & 0.48 \\
\hline
\end{tabular}

Notes: ${ }^{\dagger} A V P$ images were available for 57 affected eyes (48 eyes in the BCVA at recovery $\geq 20 / 200$ group and 9 eyes in the BCVA at recovery <20/200 group), and CNS MRI scans were available for $6 \mathrm{I}$ affected eyes (52 eyes in the BCVA $\geq 20 / 200$ at recovery group and 9 eyes in the BCVA at recovery $<20 / 200$ group). *Statistically significant ( $<<0.05$ ).

Abbreviations: NMOSD-ON, neuromyelitis optica spectrum disorder-related optic neuritis; ON, optic neuritis; NMOSD, neuromyelitis optica spectrum disorder; $n$, number; min, minimum; max, maximum; AQP4, aquaporin-4; SLE, systemic lupus erythematosus; CF, counting fingers; logMAR, logarithm of the minimum angle of resolution; AVP, anterior visual pathway; CNS, central nervous system; MRI, magnetic resonance imaging; IVMP, intravenous methylprednisolone; BCVA, best-corrected visual acuity. 
Table 5 Univariate Analysis

\begin{tabular}{|c|c|c|c|}
\hline Prognostic Factors & OR & $95 \% \mathrm{Cl}$ & p-value \\
\hline $\begin{array}{l}\text { Time from initial ocular symptoms (pain and/or visual loss) to IVMP in each affected eye } \\
\text { - Less than } 2 \text { I days }\end{array}$ & 8.39 & I.8, 38.9 & $0.007^{*}$ \\
\hline Time from initial ocular symptoms (pain and/or visual loss) to IVMP in each affected eye (days) & 0.94 & $0.9,1$ & $0.016^{*}$ \\
\hline BCVA at nadir better than $\mathrm{CF}$ & 8.00 & $0.97,1.04$ & $0.04 *$ \\
\hline
\end{tabular}

Note: *Statistically significant $(p<0.05)$.

Abbreviations: OR, odds ratio; $\mathrm{Cl}$, confidence interval; IVMP, intravenous methylprednisolone; $\mathrm{BCVA}$, best-corrected visual acuity; CF, counting fingers.

Table 6 Multivariate Analysis

\begin{tabular}{|l|l|l|l|}
\hline Prognostic Factors & OR & $\mathbf{9 5 \%} \mathbf{C l}$ & $\mathbf{P}$-value \\
\hline $\begin{array}{l}\text { Time from initial ocular symptoms (pain and/or visual loss) to IVMP in each affected eye } \\
\bullet \quad \text { Less than 2I days }\end{array}$ & 10.73 & $1.91,60.01$ & $0.007^{*}$ \\
\hline BCVA at nadir better than CF & 10.43 & $1.04,104.45$ & $0.046^{*}$ \\
\hline
\end{tabular}

Note: *Statistically significant $(\mathrm{p}<0.05)$.

Abbreviations: OR, odds ratio; $\mathrm{Cl}$, confidence interval; IVMP, intravenous methylprednisolone; BCVA, best-corrected visual acuity; CF, counting fingers.

\section{Discussion}

Records from 63 NMOSD-ON-affected eyes of 50 patients were retrospectively reviewed in our study. The aim of our study was to identify factors that predicted good visual outcomes following the first episode of NMOSD-ON in each affected eye in the Thai population.

Our cohort was similar to several previous studies in terms of AQP4 serostatus, female predominance, age at first episode of $\mathrm{ON}$ in each affected eye, laterality. ${ }^{1,10,15-24}$ Most of the affected eyes (37 eyes, 58.6\%) in our study presented with BCVA at nadir $<20 / 400$. This result was fairly similar to several other studies, which provides additional evidences that NMOSD-ON is typically severe. ${ }^{16,23}$ We found that the orbital optic nerve was the most-commonly affected segment of the AVP, based on enhancement of MRI. Two-thirds (66.6\%) of affected eyes demonstrated two or more consecutive segments of AVP involvement. These radiological findings support the finding that longitudinally extensive lesions of the retrobulbar optic nerve are commonly observed in NMOSD-ON. ${ }^{9}$ Involvement of the optic chiasm (10.5\%) and optic tract (5.2\%) were slightly lower in our study than in previous studies..$^{23,24}$ This might be due to the differences in time from initial ocular symptoms (pain and/or visual loss) to MRI examination among studies, which could affect the appearance of enhancement on MRI.

We found that, after acute treatment with IVMP, nearly all of the affected eyes (95.2\%) had improved BCVA at recovery. Median BCVA at recovery was $0.3 \log$ MAR (range 0.02.7), which was significantly better than BCVA at nadir (2.6
$\operatorname{logMAR}$, range $0.18-2.9, p<0.001)$. More than half of the affected eyes $(66.6 \%)$ showed BCVA at recovery in the range of $20 / 20$ to $20 / 70$. In our study, BCVA results at recovery were better than those in previous studies. ${ }^{10,16}$ This might be due to the fact that we assessed BCVA at recovery following only the first episode, in contrast to other studies, which evaluated final BCVA. Moreover, a large number of affected eyes in our study started treatment with IVMP very early (median time of 4 days).

We found a statistically significant correlation between time from initial ocular symptoms to start of IVMP, and visual outcome. Starting IVMP within 21 days of NMOSD-ON onset was significantly related to good visual outcome (BCVA $\geq 20 / 200$, OR 10.73, 95\% CI 1.91, 60.01, $p=0.007)$. This finding is comparable to results in a study reported by Akaishi et al, who demonstrated that a shorter duration from NMOSD-ON onset to initiation of IVMP significantly affected the visual outcome 1 year later. ${ }^{25}$ However, Akaishi et al did not state the cut-off duration from NMOSD onset to initiation of IVMP (days) that statistically significantly predicted good visual outcomes. There likely are advantages to initiating treatment with IVMP as soon as possible. Inflammatory demyelination of the optic nerve, causing perivascular cuffing, nerve sheath oedema and myelin break down, are found in the acute phase of the disease. ${ }^{6}$ Early treatment with IVMP rapidly helps control inflammation and prevents further, irreversible structural damage of the retinal nerve fiber layer. 
We also discovered that BCVA at nadir was associated with visual outcomes. BCVA at nadir that was better than $\mathrm{CF}$ was statistically significantly associated with good visual outcomes (OR 10.43, 95\% CI 1.04, 104.45, $p=0.046$ ). To our knowledge, ours is the first study to report this correlation in NMOSD-ON-affected eyes. Hansapinyo et al reported that initial BCVA of 20/20 - 20/60 was associated with good final visual outcome (their definition was final BCVA $\geq 20 / 60$ ). ${ }^{10}$ However, they included a variety of types of ON (not exclusively NMOSD-ON). Moreover, they assessed final BCVA, unlike BCVA at recovery following the first episode of ON, as in our study. Severity of BCVA at nadir might reflect the degree of structural and functional damage from the NMOSD-ON. Therefore, we hypothesized that good BCVA at nadir could relate to good BCVA at recovery; our findings support that hypothesis.

In multivariate analysis, time from initial ocular symptoms to IVMP, less than 21 days, and BCVA at nadir better than CF were both significantly associated with good visual outcomes. Therefore, if a patient's BCVA at nadir is better than CF in the affected eye, and administration of IVMP begins within 21 days of ON onset, the odds are favourable that the affected eye will recover to a good visual outcome at recovery. This finding may help clinicians in advising patients regarding their visual prognosis following a first episode of NMOSD-ON in their eye.

In our study, we found that younger age at first episode of $\mathrm{ON}$ in each affected eye tended to be associated with worse visual outcome. However, this finding was not statistically significant (OR 1.0, 95\% CI 0.97, 1.04, $p=0.92$ ). This is fairly similar to one previous study, reported by Kitley et al, ${ }^{8}$ in which younger subjects suffered from more severe disease, leading to worse visual outcomes. We did not find a significant association between co-existing autoimmune diseases and visual outcome, which is a similar result to that reported by Akaishi et al. ${ }^{25}$ In contrast to our study, some previous studies demonstrated worse visual prognosis in subjects with older age at $\mathrm{ON}$ onset and co-existing autoimmune diseases.${ }^{7,9}$ This might be due to the difference between studies in inclusion criteria, in terms of age at $\mathrm{ON}$ onset. In our study, we included 12 affected eyes (19\% of total affected eyes) of 6 pediatric patients ( $12 \%$ of all patients). Moreover, this could affect the study results since autoimmune diseases are very rare in the pediatric population.

A prior report by Akaishi et al suggested that abnormal axial signal length of the optic nerve in the acute phase significantly affected visual prognosis. ${ }^{9}$ We did not find a significant association between number of segments of
AVP involved and visual outcomes, like Akaishi's study. ${ }^{9}$ However, in our study, we investigated only the presence of two or more consecutive segments involved, based on contrast enhancement of AVP, instead of the actual length of contrast enhancement.

There were several strengths in our study. First, we collected various aspects of demographic data, and clinical and MRI characteristics, to analyse as many potential prognostic factors as possible that might affect the visual outcome. Second, the numbers of subjects and affected eyes in our study were quite large. Third, AVP and CNS MRI images were available for nearly all of the affected eyes. Lastly, pediatric subjects were also included in our study, so our results are applicable to the pediatric population.

There were, however, some limitations in our study. First, it was retrospective in design, which resulted in some incomplete data. Second, we evaluated visual outcomes following only the first episode of NMOSD-ON in each affected eye, not final visual outcomes. Third, since all patients in our study were Asian, our results might not be applicable to other ethnicities. Fourth, we did not assess other elements of visual function, such as tests of colour vision, contrast sensitivity and visual field, or structural characteristics, such as retinal nerve fiber layer thickness and macular ganglion cell analysis. Finally, we did not evaluate the adjunctive effect of PLEX, due to the small number of eyes of patients who received PLEX in our study (4 affected eyes). Further prospective study, in a larger cohort, is therefore warranted, and would provide more-conclusive information regarding prognostic factors for visual outcome following the first episode of NMOSD-ON.

\section{Conclusions}

In summary, most patients with an NMOSD-ON-affected eye, or eyes, presented with severe visual loss. Early treatment with IVMP could preserve better vision. BCVA at nadir that was better than CF and early treatment with IVMP (within 21 days of NMOSD-ON onset) were important predictors of good visual outcomes following the first episode of NMOSD-ON in each affected eye.

\section{Consent for Publication}

Not applicable.

\section{Acknowledgments}

The authors would like to thank Mr. Nattawut Unwanatham, Department of Clinical Epidemiology and Biostatistics, Faculty of Medicine Ramathibodi Hospital, Mahidol 
University, Bangkok, Thailand for his advice on the statistical analyses. We thank Claire Barnes, PhD, from Edanz Group (https://en-author-services.edanzgroup.com/ac) for editing a draft of this manuscript.

\section{Funding}

There is no funding to report.

\section{Disclosure}

The authors declare that they have no competing interests.

\section{References}

1. Sellner J, Boggild M, Clanet M, et al. EFNS guidelines on diagnosis and management of neuromyelitis optica. Eur $J$ Neurol. 2010;17:1019-1032. doi:10.1111/j.1468-1331.2010.03066.x

2. Trebst C, Jarius S, Berthele A, et al. Update on the diagnosis and treatment of neuromyelitis optica: recommendations of the Neuromyelitis Optica Study Group (NEMOS). $J$ Neurol. 2013;261:1-16.

3. Sherman E, Han M. Acute and chronic management of neuromyelitis optica spectrum disorder. Curr Treat Options Neurol. 2015;17(11):48. doi:10.1007/s11940-015-0378-x

4. Roed HG, Langkilde A, Sellebjerg F, et al. A double-blind, randomized trial of IV immunoglobulin treatment in acute optic neuritis. Neurology. 2005;64:804-810. doi:10.1212/01.WNL.0000152873.82 631.B3

5. Ghezzi A, Bergamaschi R, Martinelli V, et al. Clinical characteristics, course and prognosis of relapsing devic's neuromyelitis optica. $J$ Neurol. 2004;251:47-52. doi:10.1007/s00415-004-0271-0

6. Wingerchuk D, Hogancamp W, O'Brien P, et al. The clinical course of neuromyelitis optica (Devic's syndrome). Neurology. 1999;53:1107-1114. doi:10.1212/WNL.53.5.1107

7. Wingerchuk D, Weinshenker B. Neuromyelitis optica: clinical predictors of a relapsing course and survival. Neurology. 2003;60:848853. doi:10.1212/01.WNL.0000049912.02954.2C

8. Kitley J, Leite M, Nakashima I, et al. Prognostic factors and disease course in aquaporin- 4 antibody-positive patients with neuromyelitis optica spectrum disorder from the United Kingdom and Japan. Brain. 2012;135:1834-1849. doi:10.1093/brain/aws109

9. Akaishi T, Nakashima I, Takeshita T, et al. Lesion length of optic neuritis impacts visual prognosis in neuromyelitis optica. $J$ Neuroimmunol. 2016;293:28-33. doi:10.1016/j.jneuroim.2016.02.004

10. Hansapinyo L, Vivattanaseth C. Clinical characteristics, treatment outcomes and predictive factors in optic neuritis. Open $J$ Ophthalmol. 2018;12:247-255. doi:10.2174/1874364101812010247
11. Wingerchuk D, Banwell B, Bennett J, et al. International consensus diagnostic criteria for neuromyelitis optica spectrum disorders. Neurology. 2015;85:177-189. doi:10.1212/WNL.0000000000001729

12. Song H, Zhou H, Yang M, et al. Different characteristics of aquaporin-4 and myelin oligodendrocyte glycoprotein antibody-seropositive male optic neuritis in China. J Ophthalmol. 2019;2019:1-7. doi: $10.1155 / 2019 / 1429548$

13. Grover S, Fishman G, Anderson R, et al. Visual acuity impairment in patients with retinitis pigmentosa at age 45 years or older. Ophthalmology. 1999;106(9):1780-1785. doi:10.1016/S0161-6420 (99)90342-1

14. McAnany J, Genead M, Walia S, et al. Visual acuity changes in patients with leber congenital amaurosis and mutations in CEP290. JAMA Ophthalmol. 2013;131(2):178-182. doi:10.1001/2013. jamaophthalmol.354

15. Bruscolini A, Sacchetti M, La Cava M, et al. Diagnosis and management of neuromyelitis optica spectrum disorders - An update. Autoimmun Rev. 2018;17:195-200. doi:10.1016/j.autrev.2018.01.001

16. Merle H, Olindo S, Jeannin S, et al. Treatment of optic neuritis by plasma exchange (add-on) in neuromyelitis optica. Arch Ophthalmol. 2012;130:858-862. doi:10.1001/archophthalmol.2012.1126

17. Zhou Y, Zhong X, Shu Y, et al. Clinical course, treatment responses and outcomes in Chinese paediatric neuromyelitis optica spectrum disorder. Mult Scler Relat Dis. 2019;28:213-220. doi:10.1016/j. msard.2018.12.038

18. Wang J, Zhou H, Qin L, et al. Optic neuritis in the older Chinese population: a 5-year follow-up study. J Ophthalmol. 2017;2017:1-7.

19. Chitnis T, Ness J, Krupp L, et al. Clinical features of neuromyelitis optica in children: US network of pediatric MS centers report. Neurology. 2016;86:245-252. doi:10.1212/WNL.0000000000002283

20. Hollen C, Suhaib O, Farrow A, et al. Elderly-onset neuromyelitis optica spectrum disorder with pre-existing prednisone allergy. Case Rep Neurol. 2018;10:25-28. doi:10.1159/000485120

21. Petzold A, Pittock S, Lennon V, et al. Neuromyelitis optica-IgG (aquaporin-4) autoantibodies in immune mediated optic neuritis. $J$ Neurol Neurosurg Psychiatry. 2009;81:109-111. doi:10.1136/ jnnp.2008.146894

22. Kleiter I, Gahlen A, Borisow N, et al. Neuromyelitis optica: evaluation of 871 attacks and 1153 treatment courses. Ann Neurol. 2016;79:206-216. doi:10.1002/ana.24554

23. Srikajon J, Siritho S, Ngamsombat C, et al. Differences in clinical features between optic neuritis in neuromyelitis optica spectrum disorders and in multiple sclerosis. Mult Scler J Exp Transl Clin. 2018;4 (3): $1-12$.

24. Ramanathan S, Prelog K, Barnes E, et al. Radiological differentiation of optic neuritis with myelin oligodendrocyte glycoprotein antibodies, aquaporin-4 antibodies, and multiple sclerosis. Mult Scler. 2015;22:470-482.

25. Akaishi T, Takeshita T, Himori N, et al. Rapid administration of highdose intravenous methylprednisolone improves visual outcomes after optic neuritis in patients with AQP4-IgG-positive NMOSD. Front Neurol. 2020;11:932. doi:10.3389/fneur.2020.00932
Clinical Ophthalmology

\section{Publish your work in this journal}

Clinical Ophthalmology is an international, peer-reviewed journal covering all subspecialties within ophthalmology. Key topics include: Optometry; Visual science; Pharmacology and drug therapy in eye diseases; Basic Sciences; Primary and Secondary eye care; Patient Safety and Quality of Care Improvements. This journal is indexed on PubMed
Central and CAS, and is the official journal of The Society of Clinical Ophthalmology (SCO). The manuscript management system is completely online and includes a very quick and fair peer-review system, which is all easy to use. Visit http://www.dovepress.com/ testimonials.php to read real quotes from published authors. 